\title{
Vulnerabilidad en el transporte público: análisis cuantitativo de percepciones sobre movilidad segura
}

\author{
Vulnerability in public transport: A quantitative analysis of perception on safe mobility
}

Fidel González-Quiñones*, Claudia Patricia Contreras * y Claudia Rocío Jurado Rodríguez*

Recibido: 26 de mayo de 2018

Aceptado: 11 de diciembre de 2018

\section{Resumen}

El transporte público es un factor de suma importancia para el desarrollo y la competitividad de cualquier ciudad. El presente artículo tiene como objetivo analizar cuantitativamente las percepciones de usuarios del transporte público de la Ciudad de Chihuahua, México durante un estudio de movilidad segura realizado en el 2015, tomando en cuenta dos polígonos con alta incidencia delictiva. Fueron elegidas aquellas variables donde se podían establecer diferencias y similitudes entre hombres y mujeres, dividiendo el análisis en tres grupos: las relacionadas con la vía de acceso al transporte, con el periodo que dura el viaje y un con otras variables también de importancia. Los cuestionarios fueron aplicados a 776 personas de forma aleatoria en diversos horarios y días de la semana. Los resultados permiten establecer importantes conclusiones de acuerdo con diversas variables: Las mujeres usuarias del transporte en estas zonas violentas se muestran más temerosas, acosadas y vulnerables al usar el transporte público; sin embargo, son los hombres quienes manifiestan más incidentes reales de violencia física. Se presentan también algunas consideraciones relacionadas con la dinámica de las encuestas y la relación entre encuestadores y encuestados.

Palabras clave: grupos vulnerables, miedo, movilidad segura, transporte público.

\begin{abstract}
Public transport is a very important factor for the development and competitiveness of any city. The objective of this article is to quantitatively analyze the perceptions of public transport users in the City of Chihuahua, Mexico during a study of safe mobility carried out in 2015 , considering two geographic areas with high criminal incidence. For the study those variables where differences and similarities between men and women could be established were chosen, dividing the analysis into three groups: those related to the access route to transport, those related to the period of the trip and the third group with other variables also of importance. Questionnaires were randomly applied in different hours and days to the citizens who accepted to answer them, having a sample of 776 people. They were applied at bus stops. Results allow establishing important conclusions according to various variables. In general, women who are users of transport in these high criminal incidence zones feel fearful, harassed and vulnerable when using public transport; however, men display more physical violence incidents. Some important considerations related to the dynamics of the surveys and the relationship between interviewers and respondents are included the end.
\end{abstract}

Keywords: fear, safe mobility, public transport, vulnerable groups.

* Filiación: Universidad Autónoma de Chihuahua, Chihuahua, México. Contacto: fgonzalez@uach.mx

Cómo citar: González-Quiñones, F., Contreras, C.P. y Jurado Rodríguez, C.R. (2018). Vulnerabilidad en el transporte público: análisis cuantitativo de percepciones sobre movilidad segura. Revista de Urbanismo, 39, 1-14.

https://doi.org/10.5354/0717-5051.2018.49399 


\section{Introducción}

El transporte público es un factor de suma importancia para el desarrollo y la competitividad de cualquier ciudad. Así lo refieren Cabrera-Arana, Velásquez-Osorio y OrozcoArbeláez (2015, p. 430, cuando citan: "La movilidad es el mayor desafío de la vida urbana contemporánea". Su estructura, funcionamiento y operación se encuentran íntimamente relacionados con la calidad de vida de sus usuarios, e incluso también de aquellos que no son usuarios, ya que cuentan con cualquier otro medio para movilizarse de un punto a otro en la ciudad. Estos últimos se ven afectados indirectamente por la puntualidad y estado de ánimo de los primeros. En este sentido, Cabrera (2014, p. 19) agrupa a la movilidad y la seguridad ciudadana indicando que son "aspectos clave para garantizar mejores condiciones de accesibilidad a los bienes y servicios urbanos". Indica también que estas categorías tan relacionadas entre sí son relevantes en el desarrollo urbano y la planeación de la infraestructura para el transporte en la ciudad.

Considerando lo anterior, se realizó una investigación de enfoque cuantitativo cuyo objetivo general fue analizar las percepciones de usuarios del transporte público de la Ciudad de Chihuahua, México, tomando en cuenta dos polígonos con alta incidencia delictiva. Además, se establecieron los siguientes objetivos específicos:

1. Determinar cómo perciben los ciudadanos de las zonas seleccionadas las diversas condiciones relacionadas con el sistema de transporte público.

2. Identificar si existen diferencias en esas percepciones de acuerdo con variables de segmentación como sexo o edad.

3. Identificar si existe un perfil de los usuarios en cuanto al uso del servicio en factores como el tiempo del viaje, tiempo de espera en los paraderos y cantidad de días que usa el transporte público.

4. Analizar el número de usuarios que han sufrido violencia física o acoso durante el viaje o en el traslado al paradero

5. Inferir cuantos usuarios viajan con miedo.

La hipótesis de esta investigación es que existen diferencias importantes entre las percepciones de los usuarios analizados con relación a las condiciones de movilidad en el transporte público de acuerdo con la edad y el sexo. A su vez, la importancia de este trabajo se debe a que el tiempo y costo de traslado al trabajo, a la escuela o incluso visitar áreas de recreación se resta al tiempo libre de calidad para realizar otras actividades necesarias, como trabajar, estudiar, proveer en el hogar o incluso el ocio y, como se establece en El Programa de Naciones Unidas para los Asentamientos Humanos (ONU-Hábitat en inglés)

Una adecuada planificación urbana, para transformar nuestras ciudades en espacios sustentables y conectados, garantizará el ejercicio efectivo de los derechos sociales de todos los mexicanos y disminuirá, significativamente, las brechas de desigualdad en el país (Organización de las Naciones Unidas-Hábitat [ONU-Hábitat], 2015, p. 8)

Por sentencias como la anterior, los investigadores interesados en este tema realizan estudios del transporte público tomando en cuenta siempre a los grupos vulnerables, ya sean ciudadanos que viven en periferias, grupos étnicos, adultos mayores, entre otros. Estos estudios se realizan en el contexto del término movilidad, la cual es definida como "Una práctica social de desplazamiento diario a través del tiempo y del espacio urbano que permite el acceso a actividades, personas y lugares." (Jirón, Lange, y Bertrand, 2010, pág. 24). Adicionalmente, el autor complementa su definición aclarando que el concepto de movilidad incluye también las condiciones sociales que involucran "dimensiones espaciotemporales, corporales, interaccionales, simbólicas y afectivas, desigualmente vividas en relación con la clase, el género, la edad y la etnia" (Segura, 2014, p.193 citado por Chaves, Segura, Speroni, \& Cingolani, 2017, p. 45)

Otros autores que complementan el concepto anterior (Díaz y Jiménez, 2003), señalan que, la movilidad en la ciudad "es una relación entre muy diferentes aspectos de los roles y relaciones de género, del urbanismo y de la vida cotidiana" (citado en Zúñiga Elizalde, 2014).

La diferencia existente entre movilidad y transporte es también señalada por Gutiérrez (2012) explicando que aunque ambos términos aluden al desplazamiento de un lugar a otro, como se comprobó líneas atrás, “...la distinción apunta a entender la movilidad urbana como una performance [espacio social producido y organizado 
por una sociedad en una situación determinada] en el territorio, y al transporte como el medio o vector que realiza el desplazamiento" (p. 65). Así pues, transporte se refiere al modo de desplazamiento, ya sea un automóvil propio, transporte público,etc., y movilidad al acto, donde se involucran los aspectos sociales.

En una ciudad, las personas deberían poder movilizarse y desplazarse libremente bajo condiciones óptimas que incluyan variables diversas relacionadas con el transporte como el cuidado al medio ambiente, el respeto de choferes y usuarios y la infraestructura del sistema de transporte que incluye camiones, paraderos y rutas. Sin embargo, según Cass, Shove y Urry (2005) “los sistemas de transporte pueden llegar a ser considerados deficientes, pero no necesariamente la principal barrera de accesibilidad" (citado en Jiron y Mansilla, 2013, p. 54), ya que fallan en los criterios anteriormente mencionados desde la expulsión desenfrenada de dióxido de carbono hasta paraderos en mal estado que no generan confianza para el usuario.

Según el Instituto de Políticas para el Transporte y el Desarrollo (ITDP por sus siglas en inglés), fundado en 1985, en México es "difícil encontrar la norma que obligue al Estado a garantizar el derecho al transporte y no existe un estándar que nos permita saber cuándo este derecho se está cumpliendo y cuando no" (Instituto de Políticas para el Transporte y el Desarrollo [ITDP], 2017, p. 7). Este derecho no está estipulado en la Constitución y se encuentra apenas esbozada en el ámbito internacional de los derechos humanos.

En la discusión se aportan factores positivos como el caso del mismo ITDP (2017, pág. 8), citando algunos documentos legales que contemplan entre sus objetivos a mediano y largo plazo la necesidad de implementar un transporte de carácter sostenible. Estos son: Declaración de Río sobre el Medio Ambiente y Desarrollo (principios 1, 3, 4, 5); El Programa 21 de la ONU (capítulos 3, 6,7, 9) y el Protocolo de Kioto (Artículo 2, VII, VIII)".

Una de las asociaciones internacionales que estudian el tema del transporte público es la Asociación Internacional de Transporte Público (UITP por sus siglas en francés.) (Asociación Internacional del Transporte Público, 2017). Esta asociación ha desarrollado criterios y propuestas orientadas a garantizar una movilidad sostenible basada en la regulación de los permisos del uso de suelo, restringiendo el uso del vehículo privado y logrando un sistema de transporte público eficaz. De esta forma se concibe a la movilidad como un derecho humano, "vinculado a derechos colectivos de gran relevancia en la vida contemporánea como son: medio ambiente, espacio público y accesibilidad universal" (ITDP, 2017, p. 9)

Continuando con este concepto social, se cita a Cabrera-Arana et al. (2015, p. 430) quienes identifican dos dimensiones que son básicas en la movilidad: debe ser segura y sostenible. "Segura para que personas y objetos no estén expuestos a riesgos previsibles evitables. Sostenible para un desplazamiento con propósito, eficiente, al mínimo costo, intergeneracionalmente disponible ahora o mañana y aceptable para individuos y sociedades"

Según Rodríguez Vignoli (2008): "la movilidad cotidiana es un asunto relevante para las personas, porque una buena parte de la población es móvil"; así pues, si se viera al transporte público como un Derecho Humano, se podría considerar no solamente como un bien económico, sino como un bien social y cultural. Este derecho debe ser ejercido de manera responsable, puntual y en armonía con el medio ambiente para ser disfrutado por las generaciones actuales y futuras. De esta forma, quien tiene movilidad mejora sus posibilidades de acceder al mercado laboral, a tener una vivienda, a la educación, la cultura y el ocio. "En cierto sentido este derecho a la movilidad es una precondición de los otros derechos" (Ascher, 2005 citado por Hernández, 2012)

Además de estas condiciones sociales, en las grandes ciudades, el transporte también puede ser una estrategia determinada en parte por factores políticos, disfrazados o escondidos por otras consideraciones económicas, sociales, culturales, ecológicas e incluso de promoción turística. Estas decisiones políticas pueden condicionar otras variables del transporte como son la disponibilidad, accesibilidad, asequibilidad sin discriminación, calidad, seguridad, equidad y sostenibilidad.

Para alcanzar esa calidad, se debe dar importancia también a la movilidad insatisfactoria, un indicador presentado por Andrea Gutiérrez (2010) en su estudio Movilidad, transporte y acceso: una renovación aplicada al ordenamiento territorial, ya que muestra claros ejemplos de cuáles son los problemas que la sociedad 
reconoce en el transporte público como son las espera prologada en condiciones de intemperie, mala iluminación, higiene y falta de asientos lo cual trae consigo incomodidad o sensación de peligro y angustia durante el viaje.

En los análisis de movilidad segura es muy importante tomar en cuenta a los grupos vulnerables como señala Soto (2017, p. 128): "tanto la estructura urbana, así como los comportamientos individuales y la percepción de los espacios urbanos están de diferentes formas influenciados por factores socioculturales tales como género, edad, tipo de actividad, entre otras".

Aunque la intención inicial de esta investigación no era la de contar con una perspectiva de género, se encontró en la revisión bibliográfica una fuente importante de textos que señalaban la importancia de este factor, aún con mayor frecuencia que una perspectiva en grupos vulnerables. Entre estos artículos se cita a (Garibi, 2010: 12 citado en Dunckel-Graglia, 2013) quien afirma que es el transporte público uno de los espacios donde las mujeres sufren mayor violencia e incluso delitos sexuales no denunciados y eso "afecta sus capacidades y oportunidades de desarrollo."

La Ciudad de Chihuahua es la capital del Estado y cuenta con 878,062 habitantes, lo cual la ubica en segundo lugar estatal en cuanto a población se refiere después de la fronteriza Ciudad Juárez, que cuenta con 1,391,180 habitantes (Instituto Nacional de Estadística y Geografía, 2016). En general, la Ciudad de Chihuahua se sitúa en el lugar número 18 nacional en cuanto a su cantidad de habitantes (Organización de las Naciones Unidas-Hábitat, 2015).

Este tamaño poblacional coloca a la ciudad en un nivel considerado por la ONU-Habitat en el grupo de "zonas metropolitanas entre medianas y pequeñas" lo que hace que su infraestructura de transporte también sea más limitada en comparación con otras ciudades. En la ciudad de Chihuahua, el transporte se rige por la Ley de Transporte y sus vías de comunicación. Existe en esta ley una sola mención relacionada con las mujeres en el artículo 45 Fracción 16 que dice:
Los concesionarios y permisionarios que presten servicio público de transporte tendrán las obligaciones siguientes: ...

XVI. Contar en las unidades de transporte urbano y semiurbano, con asientos para personas con discapacidad y para mujeres con embarazo evidente, diferenciados de los demás por señalamientos o logotipos y situados junto a la puerta de acceso de las unidades de transporte, pudiendo ser ocupados por cualquier usuario, siempre y cuando no sean requeridos por alguna otra persona con discapacidad o embarazo evidente (Honorable Congreso del Estado de Chihuahua, 1994).

Al momento de realizar la presente investigación los desplazamientos se realizaban solamente en sistemas de transporte concesionado, de tipo urbano/suburbano, con unidades insuficientes y antiguas (Organización de las Naciones Unidas-Hábitat, 2015). El resto de las opciones de transporte eran el servicio de taxi, automóvil particular, a pie y el poco usado transporte personal en bicicleta.

Los habitantes de Chihuahua presentan suficientes motivos para sentir miedo tan solo por habitar en esta ciudad, considerada una de las más violentas del mundo y ubicada por el Consejo Ciudadano para la Seguridad Pública y Justicia Penal en el lugar 29 (BBC News, 2018). En el caso de las mujeres, por su condición de vulnerabilidad se puede añadir otra consideración importante para considerar que sienten temor debido al tema de los feminicidios conocido mundialmente como "Las Muertas de Juárez" en donde se ha documentado el asesinato, violación y tortura sistemática de cientos de mujeres desde el año de 1993 (Fajardo, 2018).

Este miedo y percepción de inseguridad no solamente se presenta durante la movilidad, se generaliza a diversos ámbitos como los conjuntos habitacionales (Galeana Cruz, 2018), las zonas turísticas y comerciales (Quezada, 2018) y además, se relaciona directamente con la raza, la clase, la edad y sobre todo con el género (Koskela, 2011, p. 274). El miedo prospera también cuando existen otros factores que ayudan a potencializarlo, independientemente de que el país sea o no subdesarrollado. 
Chihuahua presenta en muy alta medida al menos uno de estos factores mencionados por Pyszczek (2012, p. 42): El narcotráfico. Aunque es evidente que el miedo se encuentra presente tanto en mujeres como hombres, son las mujeres quienes generalmente deben limitar su movilidad en cierta hora y en ciertos lugares porque son peligrosos, cambiando sus rutinas y dejando de hacer sus actividades cotidianas (Rainero, 2009, p. 106).

Tabla 1.

Valores de confianza y error para determinar el tamaño de la muestra.

\begin{tabular}{ll}
\hline Descripción & Valor \\
\hline Población N= & 15,300 \\
Nivel de Confianza Z=95\% & 1,96 \\
Margen de error Se=3.5\% & 0,035 \\
Probabilidad de acierto $\mathrm{p}=50 \%$ & 0,5 \\
Tamaño muestral $n=$ & 784 \\
\hline
\end{tabular}

Fuente: Elaboración propia.

Tomando los valores anteriores, se aplicó la fórmula para calcular la muestra en poblaciones infinitas estableciendo un tamaño poblacional de 784 personas, sin embargo, en la edición y revisión final de los datos, se cancelaron por inconsistencias un total de 8 encuestas, quedando al final con una muestra válida de $n=776$.

El instrumento de medición consistió en un cuestionario con 60 variables desarrollado por el Gobierno Federal dentro del Programa Nacional de Movilidad Segura, aplicado en la totalidad del territorio nacional mexicano (Secretaría de Gobernación, 2015, p. 13) Este instrumento presenta similitudes con las variables de otros instrumentos como es el caso del Banco Interamericano de Desarrollo (Granada et. al., 2016, p. 1), donde se encuentran coincidencia de variables analizadas como la limpieza, el tiempo de espera, la seguridad, el alumbrado público, pasos peatonales, sistemas de vigilancia y respeto por el espacio vital contra los robos y el acoso.

El punto de contacto con el encuestado se estableció directamente en los paraderos de camión urbano ubicados en los polígonos con alta incidencia delictiva

\section{Metodología}

El presente estudio es cuantitativo, descriptivo, y transeccional. La zona analizada se encuentra constituida por usuarios de 20 colonias y cuenta con una población total de 15,300 personas (Instituto Municipal de Planeación Chihuahua, 2009, p.9). La muestra tomada para la presente investigación consideró los siguientes valores: 
análisis estadístico SPSS donde se analizaron los datos y se presentaron las tablas del presente artículo, con excepción de la figura 1 que se obtuvo también del Excel.

Los encuestadores fueron estudiantes de la Universidad Autónoma de Chihuahua, capacitados y supervisados estrechamente en cuanto a los aspectos metodológicos del levantamiento de las encuestas y en cuanto a sus condiciones de seguridad por tratarse de un levantamiento en zonas con alta incidencia delictiva. La capacitación se realizó por medio del Laboratorio de Auditorías de Opinión (González-Quiñones, MachinMastromatteo y Romo-González, 2018).

Debido a que los encuestados se encontraban algunas veces en el trámite de abordar un segundo autobús, se presentó un índice del $12 \%$ de cuestionarios cancelados por no haber sido respondidas en su totalidad. Existió también una cantidad no determinada de personas que se negaron a responder culpando al gobierno de las condiciones del transporte o mostrando descontento con la encuesta; sin embargo, en general, los ciudadanos accedieron al llenado de los cuestionarios sin presiones y con la aclaración del anonimato en sus respuestas.

\section{Resultados}

De los 776 encuestados, 288 fueron hombres que representan el $37 \%$ de la muestra, mientras que las mujeres encuestadas fueron 488, representando el $63 \%$. Este porcentaje de mujeres encuestadas se convierte en un dato muy significativo considerando que la mujer es quien sufre más acoso dentro y fuera del hogar. Esto no indica necesariamente que este valor esté relacionado directamente con el porcentaje del sexo de los usuarios del servicio de transporte y podría más bien estar influenciado por una predisposición de los encuestadores a elegir mujeres debido a cierta confianza en ellas. No se debe olvidar que este análisis se realizó en polígonos de alta incidencia delictiva, por lo que la apariencia física y vestuario de algunos usuarios varones podrían inconscientemente desalentar al encuestador.

El $12 \%$ de los cuestionarios se cancelaron por inconsistencias y debieron ser aplicados nuevamente pues los usuarios consideraban que no tenían tiempo de responder porque debían retirarse pronto del lugar, o debido a que la llegada del transporte que se encontraban esperando no permitiría terminar con la encuesta.

Las mujeres encuestadas presentaron un rango de los 11 a los 86 años con una media de 34.02 y una desviación estándar de 13.98 años.

En el caso de los varones, el rango resultó de 13 a 78 años con una media de 30.86 años y una desviación estándar de 14.80.

Lo datos resultaron ser muy similares entre ambos sexos, y estas medidas de dispersión y de tendencia central analizadas demuestran una amplia gama de usuarios del sistema del transporte.

Se realizó una agrupación por edades en 7 intervalos, realizando una comparación entre ambos sexos y obteniendo una diferencia interesante en cuanto el perfil de los encuestados, lo cual se observa en la siguiente gráfica: 


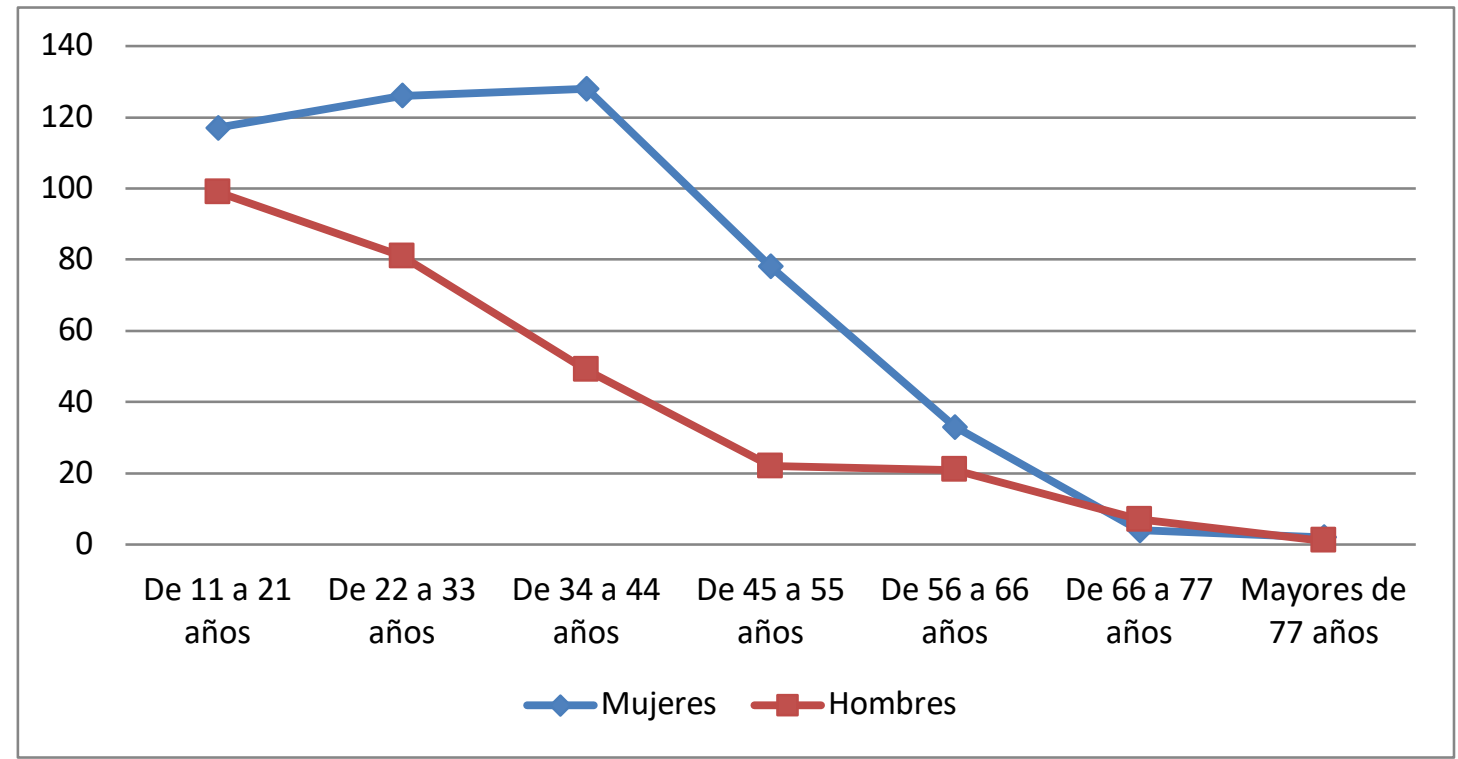

Figura 1. Distribución por edad y sexo.

Fuente: Elaboración propia.

Los datos muestran que en los hombres existe una correlación negativa perfecta entre la edad y la cantidad de entrevistados, las mujeres presentan un comportamiento muy distinto siendo las más encuestadas quienes contaban con un rango de edad de 34 a 44 años.
En cuanto a la frecuencia del uso del transporte, se encontraron datos prácticamente iguales entre ambos sexos (Tabla 2):

Tabla 2.

Frecuencia de uso del transporte público.

\begin{tabular}{lllll}
\hline & Femenino & \multicolumn{3}{l}{ Masculino } \\
\cline { 2 - 5 } Uso del transporte & Frecuencia & $\%$ & Frecuencia & $\%$ \\
\hline Diario & 391 & 80,1 & 230 & 82,1 \\
De 3 a 5 veces por semana & 67 & 13,7 & 28 & 10,0 \\
Cada 15 días & 8 & 1,6 & 8 & 2,9 \\
Al menos una vez al mes & 18 & 3,7 & 12 & 4,3 \\
Nunca & 4 &, 8 & 2 &, 7 \\
\hline Total & $\mathbf{4 8 8}$ & $\mathbf{1 0 0}$ & $\mathbf{2 8 0}$ & $\mathbf{1 0 0}$ \\
\hline
\end{tabular}

Fuente: Elaboración propia.

Se encontró que al menos el $80 \%$ de los encuestados lo utilizan a diario. Lo anterior sugiere que son usuarios que no cuentan con transporte privado. 
Tabla 3.

Tiempo de espera en el paradero

\begin{tabular}{lllll}
\hline & Femenino & \multicolumn{3}{c}{ Masculino } \\
\cline { 2 - 5 } Tiempo de espera & Frecuencia & $\%$ & Frecuencia & $\%$ \\
\hline Menos de 10 minutos & 45 & 9,2 & 29 & 10,4 \\
De 10 a 30 minutos & 224 & 45,9 & 167 & 59,6 \\
De 31 minutos a 1 hora & 170 & 34,8 & 70 & 25,0 \\
Más de una hora & 49 & 10,0 & 14 & 5,0 \\
\hline Total & $\mathbf{4 8 8}$ & $\mathbf{1 0 0}$ & $\mathbf{2 8 0}$ & $\mathbf{1 0 0}$ \\
\hline
\end{tabular}

Fuente: Elaboración propia.

En cuanto a la percepción del tiempo de espera del transporte, el 55.1\% de las mujeres tardan media hora o menos. Esta variable es diferente por quince puntos porcentuales para el caso de los hombres quienes representan el $70 \%$ en esa sumatoria de porcentajes.

Tabla 4.

Distribución del tiempo promedio de espera

\begin{tabular}{lllll}
\hline & Femenino & \multicolumn{3}{l}{ Masculino } \\
\cline { 2 - 5 } Tiempo promedio & Frecuencia & $\%$ & Frecuencia & $\%$ \\
\hline Menos de media hora & 102 & 20,9 & 89 & 31,8 \\
De 31 minutos a una hora & 193 & 39,5 & 119 & 42,5 \\
Entre una y dos horas & 157 & 32,2 & 63 & 22,5 \\
Más de dos horas & 36 & 7,4 & 9 & 3,2 \\
\hline Total & $\mathbf{4 8 8}$ & $\mathbf{1 0 0}$ & $\mathbf{2 8 0}$ & $\mathbf{1 0 0}$ \\
\hline
\end{tabular}

Fuente: Elaboración propia.

Para el caso de la percepción del tiempo del trayecto durante el transporte, nuevamente existe una diferencia de 15 puntos porcentuales entre hombres y mujeres. Las mujeres que tardan una hora o menos representaron el $60,5 \%$, mientras que el $74,3 \%$ de los varones llegan a su destino en el mismo lapso de una hora o menos. No parece existir una razón lógica para esta diferencia que indique Los hombres viven más lejos que las mujeres. Lo más lógico es pensar que, en promedio, los tiempos de distancia reales entre hombres y mujeres deberían ser los mismos. Estos valores distintos podrían tratarse más bien de una diferencia en el sentido de percepción del tiempo entre ambos sexos. Si así fuese, esta explicación podría también cuestionar el resultado de la percepción del tiempo de espera en el paradero (Tabla 5).

Para el estudio general, se establecieron preguntas dicotómicas divididas en diversas baterías. En las siguientes tablas se muestra un concentrado de las variables más significativas relacionadas con la vía de acceso al transporte, es decir el recorrido desde el hogar hasta el punto donde se encuentra la parada. $Y$ en la siguiente se muestran las variables relacionadas exclusivamente con incidentes ocurridos durante el viaje. 


\section{Variables analizadas en la ruta de acceso a la parada del} autobús

Tabla 5.

Porcentaje de incidentes en ruta de acceso distribuidos por sexo

\begin{tabular}{|c|c|c|c|}
\hline Variable & Mujeres & Hombres & Diferencia \\
\hline ¿Hay limpieza de la vía de acceso al transporte? & $50,00 \%$ & $57,10 \%$ & $-7,10 \%$ \\
\hline ¿Existen lotes o casas abandonadas en la vía de acceso? & $43,20 \%$ & $35,40 \%$ & $7,80 \%$ \\
\hline ¿Es peligrosa la vía de acceso al transporte? & $37,70 \%$ & $28,20 \%$ & $9,50 \%$ \\
\hline $\begin{array}{l}\text { ¿Has sido víctima de acoso u hostigamiento en la vía de } \\
\text { acceso? }\end{array}$ & $15,00 \%$ & $5,00 \%$ & $10,00 \%$ \\
\hline ¿Has sido insultado o amenazado en la vía de acceso? & $8,40 \%$ & $9,60 \%$ & $-1,20 \%$ \\
\hline ¿Has sido víctima de asalto en la vía de acceso? & $9,00 \%$ & $12,10 \%$ & $-3,10 \%$ \\
\hline ¿Te han agredido físicamente en la vía de acceso? & $3,50 \%$ & $7,50 \%$ & $-4,00 \%$ \\
\hline
\end{tabular}

Fuente: Elaboración propia.

En general, solo el $50 \%$ de los encuestados considera que la ruta de acceso al transporte se encuentra limpia. En la misma vía se reporta la presencia de lotes o casas abandonadas por un $35,4 \%$ de los hombres y por un $43,2 \%$ de las mujeres. La diferencia es mayor al $7 \%$ y este factor es relevante porque la presencia de estos lugares podría estar asociada a lugares donde es factible sufrir algún ataque.

En la percepción general sobre la peligrosidad de la vía de acceso, nuevamente las mujeres se muestran con porcentajes superiores a los hombres, esta vez con un 9.5\% de diferencia. Aunque esta diferencia es notable, también lo son ambos porcentajes que nos indican en

\section{Variables analizadas dentro del trayecto del transporte}

Tabla 6.

Porcentaje de incidentes en trayecto distribuidos por sexo

\begin{tabular}{llll}
\hline Variable & Mujeres & Hombres & Diferencia \\
\hline ¿Has sufrido caídas en el transporte? & $23,40 \%$ & $16,40 \%$ & $7,00 \%$ \\
$\begin{array}{l}\text { ¿Has sido víctima de acoso u hostigamiento en el } \\
\text { transporte? }\end{array}$ & $9,60 \%$ & $5,70 \%$ & $3,90 \%$ \\
$\begin{array}{l}\text { ¿Te han insultado o amenazado en el transporte? } \\
\text { ¿Te han asaltado en el transporte? }\end{array}$ & $4,70 \%$ & $8,60 \%$ & $-3,90 \%$ \\
¿Has sido agredido físicamente en el transporte? & $4,70 \%$ & $7,10 \%$ & $-2,40 \%$ \\
\hline
\end{tabular}

Fuente: Elaboración propia como peligrosa.

En el caso del acoso u hostigamiento, el $5 \%$ de los hombres lo manifestaron contra el $15 \%$ de las mujeres. Nuevamente son las mujeres quienes presentan los mayores porcentajes en cuanto a las percepciones negativas.

Las últimas tres variables analizadas que son los insultos, asalto y agresiones físicas presentan una inversión en los porcentajes pues son ahora los hombres quienes lo manifiestan en mayor medida, aunque las diferencias entre los porcentajes son menores. 
El 23,4\% de las mujeres reporta haber sufrido caídas durante el transporte. Este porcentaje resulta 7\% menor para el caso de los hombres. Este es otro factor de riesgo donde las mujeres parecen más vulnerables.

Cuando se preguntó a los usuarios si el transporte había sufrido algún accidente, el porcentaje resultó prácticamente el mismo entre hombres y mujeres (del $17 \%$ al $18 \%)$, lo cual es de esperarse en una pregunta donde no debería existir diferencia por el sexo; sin embargo, se encontró que mientras el usuario es más joven, en porcentaje, más reporta haber sufrido accidentes. Lo anterior se observa en la siguiente tabla:

Tabla 7.

Distribución por rango de edad de usuarios que respondieron si habían sufrido algún accidente en el transporte

\begin{tabular}{ll}
\hline Rango de edad & $\%$ \\
\hline De 11 a 21 años & 22,7 \\
De 22 a 33 años & 18,8 \\
De 34 a 44 años & 16,4 \\
De 45 a 55 años & 15,0 \\
De 56 a 66 años & 11,1 \\
De 66 a 77 años & 9,1 \\
Mayores de 77 años & 0,0 \\
\hline
\end{tabular}

Fuente: Elaboración propia

Una de las preguntas más importantes en este estudio cuestionó si el usuario había sido víctima de acoso u hostigamiento durante el transporte y el porcentaje resultó ser relativamente bajo en ambos casos, pero un $4 \%$ superior para las mujeres, alcanzando casi un $10 \%$ de las encuestadas.
En el caso de los insultos, asaltos y agresiones físicas, nuevamente son los varones quienes reportan estas situaciones casi el doble de las ocasiones reportadas por las mujeres. Estos porcentajes en general, aunque podrían parecer bajos, demuestran que el transporte público en general no es seguro.

\section{Otras variables analizadas}

Tabla 8.

Distribución de porcentajes por sexo de otras variables

\begin{tabular}{llll}
\hline Variable & Mujeres & Hombres & Diferencia \\
\hline ¿En los alrededores del paradero que usas hay policías? & $38,10 \%$ & $46,80 \%$ & $-8,70 \%$ \\
¿Sabes a quién acudir en caso de emergencia en el transporte? & $35,00 \%$ & $38,60 \%$ & $-3,60 \%$ \\
¿Viajan separados mujeres, niños y adultos mayores de los hombres? & $3,10 \%$ & $5,70 \%$ & $-2,60 \%$ \\
¿Hay señalamientos e información sobre la ruta del conductor? & $49,40 \%$ & $48,90 \%$ & $0,50 \%$ \\
\hline
\end{tabular}

Fuente: Elaboración propia.

Para complementar el estudio se agruparon otras variables interesantes, por ejemplo, la percepción de la presencia policiaca, la cual presenta diferencia del $8,7 \%$ entre ambos sexos, siendo más reportada por los varones. La presencia policiaca real es la misma en todos los casos, pero es interesante como las mujeres lo reportan en menor medida.

Quienes manifiestan saber en mayor medida a quien acudir en caso de emergencia en el transporte son nuevamente los varones, con un $3,6 \%$ por encima de las 
mujeres, lo cual nuevamente abona a la percepción de indefensión que es ligeramente mayor en las mujeres.

En la Ciudad de Chihuahua no existe la costumbre de otras ciudades de México donde se separan los hombres y mujeres durante las horas pico del transporte, sin embargo, el $5,7 \%$ de los hombres consideran que las mujeres y niños viajan separados de los hombres. Por costumbre estas separaciones se realizan entre las partes frontal y posterior del autobús, ocupando los varones esta última. Las mujeres perciben esto solo en el 3,10\% de los casos.

En general la mitad de los encuestados manifestaron que existen señalamientos sobre la ruta del conductor. Esto es importante pues, aunque los señalamientos existan no están visibles o no son percibidos por el usuario.

\section{Discusión y Conclusiones}

Se han obtenido importantes conclusiones al analizar las diferencias entre el sexo y ciertas variables analizadas. Esto es importante porque contribuye a enriquecer las limitadas investigaciones existentes en cuanto a la relación a la movilidad en México. En este sentido, la ONUHábitat (2015, p. 35) señala que, a pesar de la amplia documentación de las diferencias en la movilidad entre hombres y mujeres, "en México existe poca información que dimensionen las necesidades y retos en cada ciudad, y en la práctica son contados los casos donde se han instrumentado políticas públicas y proyectos al respecto".

El mismo documento añade que la literatura de género y movilidad coincide en que las mujeres realizan viajes más cortos y de múltiples propósitos a sitios más dispersos, en horarios que permiten complementar el hogar y el trabajo. Estas necesidades no se toman en cuenta para diseño del transporte público de las ciudades y ni de las calles.

De la misma manera, el estudio ayuda a percibir qué tan protegidos o seguros se sienten hombres y mujeres respecto al transporte público y la dinámica que este desarrolla en relación con el espacio urbano. En este sentido, Páramo y Burbano (2011, p. 68) señalan que:

Un aspecto por abordar es la conectividad entre los lugares de vivienda privados y los lugares públicos, adoptando aspectos de diseño y otras medidas para mejorar el tránsito público o el trasporte público e incrementar la seguridad, permitiendo a las mujeres y a los demás viajar más fácilmente, más frecuentemente y durante distintos momentos del día.

La presente investigación, analiza variables muy similares a las de los dos ejemplos anteriores. Dentro de los hallazgos más importantes se puede señalar que las mujeres encuestadas perciben las diversas variables relacionadas con el transporte de forma más desventajosa que los hombres, al calificar factores como la limpieza, vía de acceso y presencia policiaca con porcentajes ligeramente inferiores a las calificaciones de los varones.

En cuanto a las agresiones reales que son insultos y asaltos, son los hombres quienes manifiestan los porcentajes más elevados, lo cual indica en general una dinámica ligeramente más violenta que en el caso de las mujeres.

Las variables que mostraron una considerable diferencia entre ambos sexos fueron las relacionadas con el tiempo, tanto de la espera del transporte como de la duración del viaje. En el primero de los casos se podría establecer la hipótesis de que esto es debido a una mayor predisposición de los hombres para abordar el autobús aun encontrándose lleno. Para el segundo caso, relacionado con el tiempo de duración del viaje la posible explicación es menos clara, y podría deberse solamente a una percepción de las mujeres relacionada con un mayor estrés durante el viaje. Es de suponerse en este caso que la mujer viaja más estresada y con miedo, con la subsecuente percepción de un mayor tiempo al realizar su trayecto.

Aunque el cuestionario elaborado no preguntaba directamente el nivel de percepción del miedo, estos últimos datos nos permiten inferir que el miedo se encuentra presente y es sentido en mayor medida por las mujeres, aún y cuando sean los hombres quienes reportan en mayor medida haber sufrido actos de violencia física real.

Existieron algunas variables que no presentaron diferencia al ser analizadas respecto al sexo, pero si se marcó una clara diferencia en cuanto a la edad. Mientras más joven es el usuario, más reportó haber sufrido accidentes durante el transporte. 
El instrumento de medición para esta investigación (realizado por el Gobierno Federal de México dentro del Programa Federal conocido como Movilidad Segura) presenta ciertas características que a los autores de esta investigación les hubiese gustado cambiar: Se realizaron previamente rangos en ciertas preguntas clave como el tiempo del viaje y tiempo de espera en el paradero, renunciando así al análisis de medidas de tendencia central y de dispersión y a los coeficientes de correlación que permiten las variables cuantitativas. Incluso en los rangos de agrupación existen errores como el que define: "de 31 minutos a una hora y de una hora a dos horas, lo cual se prestó a confusión entre los encuestados pues quienes consideraban que su tiempo de traslado era de una hora" podían responder en ambas opciones.

En los estudios de clima laboral generalmente se hace énfasis en que existe una mayor convivencia y tiempo compartido con los compañeros de trabajo que con los familiares cercanos. Para el caso de este segmento poblacional analizado, esto cobra una importancia similar sobre todo porque los encuestados manifestaron en más del $80 \%$ de los casos que utilizan el transporte público todos los días y el $90 \%$ de ellos en al menos tres días de la semana. Por desgracia, como ya se mencionó, el diseño del cuestionario no permite establecer el tiempo promedio de espera en el paradero ni el tiempo promedio del viaje, pero el análisis de contingencia de las variables analizadas permite observar que en la inmensa mayoría de los casos la suma de ambos tiempos multiplicados por dos, es decir, el trayecto de ida y el de vuelta tienen como resultado más de dos horas por día, todos los días para el $80 \%$ de los encuestados.

Según los datos poblacionales de la Ciudad de Chihuahua, existe un $49.3 \%$ de hombres y un $50.7 \%$ de mujeres (INEGI, 2015), sin embargo, los datos obtenidos revelan que se encuestó un $63 \%$ de mujeres y un $37 \%$ de hombres. (Vale recordar que, según las instrucciones del Gobierno Federal, para este cuestionario no se estratificó). Esto es muy significativo y plantea nuevas preguntas para otras investigaciones: ¿Sienten miedo también los investigadores y eligen primordialmente mujeres para encuestar? ¿Son las mujeres más propensas a responder este tipo de encuestas? ¿Las mujeres utilizan con mayor frecuencia el transporte público que los hombres? La hipótesis de que esto se debe a los horarios y días de aplicación queda descartada pues se realizó el levantamiento en diversas horas y días de la semana.

Los encuestadores reportaron una cantidad importante y no cuantificada de personas que se negaron a responder, mostrando un nivel alto de molestia y críticas al gobierno. Las razones de este comportamiento se desconocen, pero es importante puntualizar que en la zona en cuestión, caracterizada por alto índice de violencia y delincuencia, se podría tratar de individuos que se dedican a actividades ilícitas.

Durante la aplicación de los cuestionarios, frecuentemente se observaron personas vestidas de civil ejerciendo algún tipo de vigilancia sobre el proceso. La opinión general de los supervisores e investigadores del estudio es que podría tratarse de "halcones", término utilizado por los narcotraficantes locales para designar a quienes informan sobre las acciones realizadas en las zonas donde ejercen sus actividades. Como un esfuerzo para contrarrestar lo anterior, se dotó a todos los involucrados en la investigación de un chaleco de identificación con el logotipo de la Universidad Autónoma de Chihuahua (UACH) bordado de una forma muy visible, de tal manera que pudieran ser identificados en todo momento por los posibles delincuentes de la zona y de esta forma, permitirles realizar su labor sin contratiempos, aprovechando el prestigio e imagen de la $\mathrm{UACH}$ en la sociedad local.

Los datos que arroja esta investigación son trascendentales, pero carecen de utilidad si se archivan o se sacan de contexto, por ello es muy importante que sean dados a conocer en revistas científicas donde confluyan estudiantes, investigadores y urbanistas capaces de analizar, evaluar y comparar esta información con las situaciones de movilidad presentadas en otros países o zonas geográficas. En este sentido, la cooperación y el aprendizaje de soluciones tomadas en otras regiones con problemas similares son muy importantes para elevar el nivel de vida de los ciudadanos usuarios del transporte pero también de los no usuarios, pues también se involucran y les afecta directa o indirectamente la situación del transporte público, ya sea al compartir la misma infraestructura de calles y caminos o al depender de la puntualidad y tiempo de traslado de quienes no cuentan con transporte privado [B] 


\section{Referencias}

Asociación Internacional del Transporte Público. (2017). Advancing Public Transport. Obtenido de http://www.uitp.org/

BBC News. (7 de Marzo de 2018). Estas son las 50 ciudades más violentas del mundo (y 42 están en América Latina). Recuperado el 21 de Noviembre de 2018, de https://www.bbc.com/mundo/noticias-americalatina-43318108\#anchor1

Cabrera Barrios, T. (2014). MOVILIDAD COTIDIANA Y SEGURIDAD URBANA DESDE UNA PERSPECTIVA DE GÉNERO. Caso de estudio: Troncal Caracas de Transmilenio. En D. d. Catalunya (Ed.), Seminario Internacional de Investigación en Urbanismo "VI Seminario Internacional de Investigación en Urbanismo, Barcelona-Bogotá, junio 2014". Barcelona: Departament d'Urbanisme i Ordenació del Territori. Universitat Politècnica de Catalunya. Recuperado el 23 de Abril de 2018, de Universitat Politècnica de Catalunya:

https://upcommons.upc.edu/bitstream/handle/2099 $\angle 15990 / 079$ BGT Cabrera Tatiana.pdf?sequence $=1 \&$ isAllowed $=y$

Cabrera-Arana, G., Velásquez-Osorio, N. y OrozcoArbeláez, A. (2015). Movilidad: Aporte para su discusión. Revista Facultad Nacional de Salud Pública, 33(3), 429-434. https://doi.org/10.17533/udea.rfnsp.v33n3a13

Chaves, M., Segura, R., Speroni, M. y Cingolani, J. (2017). Interdependencias múltiples y asimetrías entre géneros en experiencias de movilidad cotidiana en el corredor sur de la Región Metropolitana de Buenos Aires. Revista Transporte y Territorio, 16, 41-67.

Dunckel-Graglia, A. (2013). Rosa, el nuevo color del feminismo: un análisis del transporte exclusivo para mujeres. La Ventana. Revista de estudios de género, 4(37), 148-176. Recuperado de http://www.scielo.o rg.mx/scielo.php?script=sci arttext\&pid=S140594362013000100007\&lng=es\&t/ng=es

Fajardo, G. (5 de Agosto de 2018). Las muertas de arena, las muertas de Juárez. Recuperado el 21 de Noviembre de 2018, de NEXOS, Cultura y Vida Cotidiana: https://cultura.nexos.com.mx/?p=16468
Galeana Cruz, S. (2018). Percepción de seguridad en espacios públicos de conjuntos habitacionales de interés social: Poza Rica y Coatzintla, 2000 a 2016. Revista de Urbanismo, 38, 1-14. https://doi.org/10.5 354/0717-5051.2018.47084

González-Quiñones, F., Machin-Mastromatteo, J., \& Romo-González, J. R. (17 de Abril de 2018). Preventing crime and violence through social, educational and informational interventions. Information Development, 34(3), 309-314. https://doi.org/10. $\underline{1177 / 0266666918770383}$

Granada, I., Anne-Marie, U., Monge, A., Ortíz, P., Pérez, D., Montes, L. y Caldo, A. (2016). El porqué de la relación entre género y transporte. Recuperado el 23 de Abril de 2018, de Banco Interamericano de Desarollo: https://publications.iadb.org/bitstream/handle/1131 9/7441/El-porque-de-la-relacion-entre-genero-ytransporte. $P D F$ ? sequence $=4 \&$ isAllowed $=y$

Gutiérrez, A. (2010). Movilidad, transporte y acceso: Una renovación aplicada al ordenamiento territorial. Scripta Nova, 14(86).

Gutiérrez, A. (2012). ¿Qué es la movilidad? Elementos para (re) contruir las definiciones básicas del campo del transporte. Bitácora Urbano Territorial, 21(2), 6174.

Hernández, D. (2012). Activos y estructuras de oportunidades de movilidad. Una propuesta analítica para el estudio de la accesibilidad por el transporte público, el bienestar y la equidad. EURE, 38(115), $117-$ 135. https://doi.org/10.4067/s0250-71612012000300 $\underline{006}$

Honorable Congreso del Estado de Chihuahua. (29 de enero de 1994). Ley de Transporte y sus vías de comunicación. Chihuahua, Chihuahua, México.

INEGI (2015). Número de Habitantes. Recuperado el 21 de Noviembre de 2018, de http://cuentame.inegi.org. $\mathrm{mx} /$ monografias/informacion/chih/poblacion/ 
Instituto de Políticas para el Transporte y el Desarrollo. (27 de febrero de 2017). Derecho al Transporte Público Adecuado. Obtenido de Instituto de Políticas para el Transporte y el Desarrollo: http://mexico.itdp.org/wpcontent/uploads/Mesa-5-Rodrigo-Gutie\%CC\%81rrezIIJ-UNAM.pdf

Instituto Municipal de Investigación y Planeación. (8 de febrero de 2016). Programa Municipal de Prevencion Social de la Violencia y la Delincuencia de Ciudad Juárez Chihuahua. Recuperado el 23 de Abril de 2018, de Instituto Municipal de Investigación y Planeación: http://www.imip.org.mx/Beta/pdu2016/Anexos/Prog ramaMunicipalPrevencionSocialdelaViolenciaDelincue ncia/PMPSVyD.pdf

Instituto Municipal de Planeación Chihuahua. (Agosto de 2009). PLAN DE DESARROLLO URBANO DE LA CIUDAD DE CHIHUAHUA: VISIÓN 2040. Recuperado el 19 de Noviembre de 2018, de https://implanchihuahua. gob.mx/IMPLAN-

Datos/PDU2040/pdf/PDU2040 2009/Documento/PD U2040 2009-02\%20Diagnostico.pdf

Instituto Nacional de Estadística y Geografía (2016). Panorama sociodemográfico de Chihuahua 2015. México: INEGI.

Jiron, P. y Mansilla, P. (2013). Atravesando la espesura de la ciudad: vida cotidiana y barreras de accesibilidad de los habitantes de la periferia urbana de Santiago de Chile. Revista de Geografía Norte Grande, 54, 53-74. https://doi.org/10.4067/s0718-34022013000300004

Jirón, P., Lange, C., y Bertrand, M. (2010). Exclusión y desigualdad espacial: retrato desde la movilidad cotidiana. Revista INVI, 25(68), 15-57. https://doi.org/10.4067/s0718-83582010000100002

Koskela, H. (2011). 'El desafío del miedo' - delito y miedo al delito como problemas sociales urbanos. Brazilian Geographical Journal: Geosciences and Humanities research medium, 2(2), 274-285.

Organización de las Naciones Unidas-Hábitat. (2015). Reporte nacional de movilidad urbana en México 20142015: Reporte Global en Asentamientos Humanos de ONU-Hábitat. México, D.F.: ONU-Hábitat.
Páramo, P. y Burbano Arroyo, A. (2011). Género y espacialidad: análisis de factores que condicionan la equidad en el espacio público urbano. Universitas Psychologica,10(1), 61-70.

Pyszczek, O. L. (2012). Los espacios subjetivos del miedo: construcción de la estigmatización espacial en relación con la inseguridad delictiva urbana. Cuadernos de Geografía - Revista Colombiana de Geografía, 21(1), 41-54. https://doi.org/10.15446/rcdg.v21n1.30694

Quezada, D. (2018). La gentrificación comercial y estigmatización a partir de la violencia. El caso del centro histórico de Ciudad Juárez, Chihuahua, 20092017. Revista de Urbanismo, 38, 1-16. https://doi.org/10.5354/0717-5051.2018.48272

Rainero, L. (2009). Más mujeres en la calle, sin miedo y sin violencia. En M. d. mujeres, Experiencias sobre ciudades seguras para las mujeres (pp. 104-107). Ciudad de México: Inmujeres Ciudad de México.

Rodríguez Vignoli, J. (2008). Movilidad cotidiana, desigualdad social y segregación residencial en cuatro metrópolis de América Latina. EURE, 34(101), 49-71. https://doi.org/10.4067/s0250-71612008000300003

Secretaría de Gobernación (2015). Guía para el desarrollo de proyectos de prevención social del delito con participación ciudadana. Recuperado el 23 de Abril de 2018, de SUBSEMUN 2015: http://www.secretariadoejecutivo.gob.mx/docs/pdfs/ subsemun/NORMATIVIDAD/FORMATOS CEDULAS $Y$ REPORTES/Guiadesarrolloproypscypdsubsemun2015. pdf

Soto, P. V. (2017). Diferencias de género en a movilidad urbana. Las experiencias de viaje de mujeres en el Metro de la Ciudad de México. Revista Transporte y Territorio, 16, 127-146.

Zúñiga Elizalde, M. (2014). Las mujeres en los espacios públicos: entre la violencia y la búsqueda de libertad. Región y sociedad, 4, 77-100. https://doi.org/10.22 198/rys.2014.0.a87 